\title{
Parâmetros sanguíneos e morfométricos de bovinos submetidos à prova do laço comprido
}

\author{
[Blood and morphometric parametres of beef cattle submitted to breakaway roping competitions]
}

\section{"Artigo Científico/Scientific Article"}

\author{
Dalton Mendes Oliveira*, Waldyr Castro Pereira Junior, Cleiton José Piazzon, \\ Loraine Saldanha Escobar, Marcos Gregory Dias Reis, Allan Motta Couto
}

Universidade Estadual de Mato Grosso do Sul, Unidade Universitária de Aquidauana, Aquidauana-MS, Brasil. *Autor para correspondência/Corresponding author: E-mail: dmo@uems.br

\begin{abstract}
Resumo
O estudo teve por objetivo avaliar os parâmetros sanguíneos e morfométricos em bovinos submetidos à prova de laço comprido. Foram utilizados 45 machos, Nelorados, divididos em três grupos: animais que participaram da prova do laço comprido (PLCO), animais que apenas treinaram (TREI) e animais sem atividade (SEMA). Foram realizadas coletas de sangue em sete animais escolhidos de forma aleatória de cada um dos grupos, nos meses de maio (Período 1), agosto (Período 2) e dezembro (Período 3) para análise de ureia, creatinina e proteína total. No grupo PLCO, outros sete animais foram escolhidos e submetidos a coletas de sangue durante a realização de provas do laço, para análises hematológicas. As mensurações morfométricas foram realizadas com hipômetro metálico e fita métrica flexível. Os dados foram submetidos ao programa estatístico R 2.11. As médias foram comparadas utilizando o teste de Scott Knott ao nível de 5\% de significância. A concentração de ureia foi menor nos animais do grupo PLCO, entretanto, esta diferença ocorreu apenas no Período 1, neste mesmo período a concentração de creatinina foi menor nos animais do grupo TREI. Não houve diferença para os grupos quanto as análises hematológicas. As mensurações morfométricas foram superiores no grupo PLCO comparativamente ao grupo TREI. A creatinina e a ureia não permitiram analisar alterações fisiológicas em bovinos submetidos a prova de laço comprido. O desenvolvimento corporal foi maior nos animais que sofrem o estímulo muscular durante as provas.
\end{abstract}

Palavras-chave: Creatinina; estresse; morfometria; ruminantes; ureia.

\begin{abstract}
The aim of this study was to assess blood and morphometric parameters in beef cattle submitted to roping competitions. In order for these analyses to be carried out, 45 male Zebu were divided into three groups: animals that compete; (BRCO), animals that only practiced to compete (PRAC), and animals with no activity (WACT). For the urea, creatinine and total protein analysis, blood samples were collected in the months of May (Period 1), August (Period 2) and December (Period 3) from seven animals selected randomly from each of the groups. Also for the hematological analysis, seven other animals from the BRCO group were selected and had blood sampled during the competitions. Morphometric measurements were performed with a metal hippometer and a flexible tape measure. The data were analyzed using the statistical program R 2.11. The means were compared by using the Scott Knott test at a 5\% significance level. The urea concentration was lower in the BRCO group, however, this difference occurred only in Period 1; in the same period, the creatinine concentration was smaller in the animals from the PRAC group. There was no difference for the groups regarding the hematological analyses. The morphometric measurements were superior in the BRCO group when compared to the PRAC group. Creatinine and urea did not allow for the analysis of physiological alterations in animals that compete. Body development was greater in animals that undergo muscle stimulation during the roping competitions.
\end{abstract}

Keywords: Creatinine; stress; morphometry; ruminants; urea. 


\section{Introdução}

$\mathrm{O}$ esporte do laço comprido tem um papel muito importante no estado de Mato Grosso do Sul, onde tornou-se uma tradição entre fazendas e faz parte de festividades realizadas em todo estado, no entanto, junto com essa tradição, os animais são submetidos ao estresse ambiental e alimentar que podem afetar a taxa de crescimento.

De maneira geral, o estresse alimentar pode gerar uma acentuada redução no ganho, entretanto, a partir do momento em que o animal é novamente realimentado ad libitum ocorre uma taxa de crescimento acima do normal, que se convencionou chamar de ganho ou crescimento compensatório. Este crescimento é modulado pelo resultado do aumento na síntese em relação à degradação (Van Eenaeme et al., 1998). Dessa forma, o gasto energético de um animal de produção está associado as atividades diárias de deslocamento e pastoreio (Lachica et al., 1997), no entanto, o gasto oriundo de atividades físicas pode determinar o seu desenvolvimento.

De acordo com Sejian et al. (2013) o aumento da exigência energética de animais submetidos ao exercício físico de caminhada está associado à redução no peso corporal e ganho médio diário. Ainda, Sejian et al. (2012) associaram o estresse da caminhada em ovinos a menor ingestão de alimento quando comparado ao grupo controle, ou seja, os animais que não sofreram $o$ estresse. Ademais, em garrotes submetidos ao transporte a pé por comitiva, observou-se perda de peso durante o período de caminhada (D'Oliveira et al., 2014), entretanto, após o término, o ganho de peso permaneceu baixo, indicando que o exercício pode comprometer a recuperação destes animais.

Em suma, o crescimento e o desenvolvimento são processos que ocorrem naturalmente, até que o animal atinja sua maturidade. Essas modificações fazem com que ocorram alterações que são melhores avaliadas pelo ganho de peso. Esses fatores associam-se a taxa de ganho e composição química dos tecidos depositados, sendo influenciados pelas interações hormonais, nutricionais, genéticas e, principalmente, metabólicas (Bullock et al., 1993).

Dessa forma, a concentração de metabólitos proteicos no sangue como a ureia e a creatinina tem sido empregados como indicadores do metabolismo muscular, sendo os níveis sanguíneos da creatinina pouco afetado pela dieta, sendo usado como referência para corrigir mudanças nas variações de ureia sanguínea (Gonzalez et al., 2000). Entretanto, a prática do esporte pelos animais pode gerar adaptações fisiológicas, o que pode reduzir o risco de queda de desempenho devido ao aumento da capacidade do animal a submeter-se a esforços longos e contínuos (Camargo et al., 2009), o que pode alterar o metabolismo de componentes proteicos.

Neste contexto, o estudo teve por objetivo avaliar ureia, creatinina e proteína total em bovinos submetidos à prova do laço comprido, assim como, seus parâmetros morfométricos.

\section{Material e Métodos}

\section{Local de realização e animais utilizados}

O projeto foi realizado no município de Aquidauana, Mato Grosso do Sul. Foram utilizados 45 animais não castrados, nelorados, contemporâneos, com idade inicial média de 12 meses e peso vivo inicial médio de $235,5 \mathrm{~kg}$ sendo divididos de forma aleatória em três grupos: 15 animais que participaram da prova do laço comprido (PLCO), ocorrida nos meses de maio e dezembro de 2016 e realizada durante um período de 3 dias, aos quais os animais eram mantidos em piquetes com alimentação e água disponível quando não submetidos a prova, já quando em atividade (prova) eram mantidos por um período de duas horas em curral e corriam cerca de $120 \mathrm{~m}$, entre 3 a 5 vezes; 15 animais utilizados semanalmente para treino de peões (competidores) (TREI), equivalendo a um período de 12 horas por semana ou quatro horas por dia não consecutivo durante a semana e outros 15 animais que não participavam da prova do laço e não eram utilizados no treino de peões, o chamado grupo sem atividade (SEMA). Todos os animais foram mantidos em sistema extensivo de pastejo e suplementados semanalmente, quando não envolvidos em nenhuma atividade.

\section{Coleta de Amostras e variáveis analisadas (Creatinina, Ureia e Proteína Total)}

Para a avaliação dos níveis de creatinina, ureia e proteína total (PtnT), foram realizadas três coletas de sangue por meio de venopunção da jugular em tubos sem anticoagulante de sete animais escolhidos de forma aleatória de cada um dos grupos. As determinações de creatinina, ureia e proteína total foram por método cinético, cinético-UV e colorimétrico-biureto, respectivamente. Análises bioquímicas foram 
realizadas utilizando-se kit comercial Analisa Diagnóstica (Gold Analisa Diagnóstica Ltda, Belo Horizonte, MG).

Estes animais foram mantidos nas coletas nos meses de maio (Período 1), agosto (Período 2), e dezembro (Período 3), do ano de 2016. As coletas nos meses de maio e dezembro, foram realizadas 15 dias após a realização da prova de laço comprido, para que não houvesse efeito acumulatório de metabólico na corrente sanguínea do animal, que interferissem nas análises, já no mês de agosto não houve competição. $O$ sangue foi centrifugado a $2400 \mathrm{rpm}$, durante 10 minutos para obtenção do soro, ao qual foi acondicionado em tubos Eppendorf® e estocado a $-20^{\circ} \mathrm{C}$ para posterior análise.

\section{Análise Hematólogica}

Para os animais do grupo PLCO, outros sete animais foram escolhidos de forma aleatória para análises hematológicas, sendo avaliado hematócrito, hemoglobina, hemácia, volume corpuscular médio (VCM), hemoglobina corpuscular média (HCM) e concentração de hemoglobina corpuscular média (CHCM).

As coletas de sangue nas duas competições (meses de maio e dezembro de 2016) foram realizadas nos mesmos animais. Para a análise coletaram-se duas amostras de sangue de cada animal por meio da venopunção jugular em tubos com anticoagulante para hematologia (EDTA $10 \mathrm{~g} / \mathrm{dL}$ ), uma antes e outra após a realização da prova de laço comprido ocorrida sempre no primeiro dia de competição, em seguida cada amostra de sangue foi levada para o laboratório para análise dos componentes em equipamento automático (Sysmex Kx-21N, Roche), por meio de impedância elétrica (corrente direta), fotometria e microscopia. Em todas as análises sanguíneas, as amostras foram armazenadas em um recipiente de isopor acondicionado com gelo e levado ao laboratório em um período de aproximadamente 1 hora.

\section{Mensurações morfométricas}

As mensurações morfométricas foram realizadas em intervalos de 40 dias, com início no mês de maio de 2016 e fim no mês de dezembro de 2016. Para realização das medidas corporais, os animais eram direcionados ao tronco de contenção, onde eram posicionados, evitando-se quaisquer desníveis que pudessem provocar erros nas medidas (Pacheco et al., 2008), posteriormente, eram obtidas, sempre pelo mesmo técnico (Figura 1).

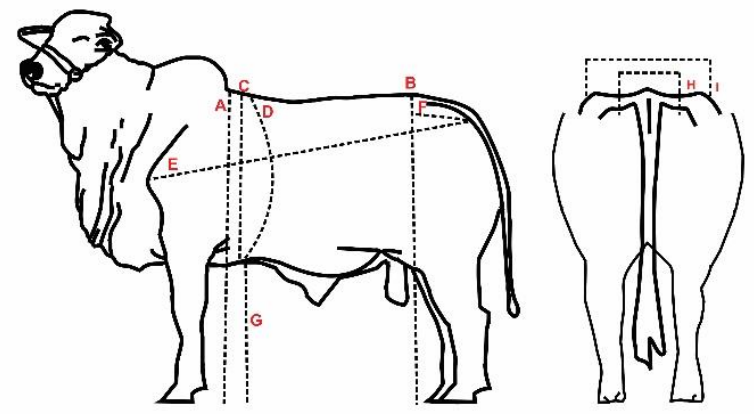

Figura 1. Medidas morfométricas. A: Altura do anterior; B: Altura do posterior; C: Perímetro torácico D: Profundidade torácica; E: Comprimento corporal; F: Comprimento de garupa; G: Altura sub esternal; H: Largura da garupa entre os ísquios; I: Largura da garupa entre os ílios.

As mensurações utilizadas foram: altura do anterior (AA): medida tomada da região da cernelha ao solo; altura do posterior (AP): medida tomada desde a região anterior do sacro ao solo; profundidade torácica (PTO): a largura máxima da região torácica na altura do arco da quinta costela; perímetro torácico (PT): pelo contorno do tórax, passando pelo cilhadouro e voltando perpendicularmente à linha do dorso; comprimento corporal (CC): medido do ponto mais cranial da articulação do úmero até o ponto mais caudal da articulação ílio-isquiática; comprimento de garupa (CG): medida da tuberosidade ilíaca externa até a ponta do ísquio; largura da garupa entre os ílios (LGI): largura máxima entre as tuberosidades dos ílios; largura da garupa entre os ísquios (LGIS): largura máxima entre as tuberosidades dos ísquios e altura sub esternal (ASE): medida da região esternal ao solo. Todas as mensurações foram realizadas com hipômetro metálico, com exceção do PT que foi medido próximo das patas anteriores na região das axilas com auxílio de uma fita métrica flexível. O peso vivo foi mensurado em balança apropriada para animais de grande porte.

Foi utilizado delineamento inteiramente casualizado. Os dados foram submetidos ao $\begin{array}{lllll}\text { programa } & \text { estatístico } & \mathrm{R} & 2.11 .0 & \text { (R }\end{array}$ DEVELOPMENT CORE TEAM, 2008). As médias foram comparadas utilizando o teste de Scott Knott ao nível de 5\% de significância.

\section{Resultados e Discussão}

A concentração de ureia foi menor nos animais do grupo PLCO, comparativamente aos outros dois grupos (Tabela 1), entretanto, tal diferença ocorreu apenas no Período 1. Durante o 
ganho compensatório é comum observar o crescimento acelerado dos animais o qual é modulado pelo aumento na síntese em relação à degradação, o que resulta em melhoria na deposição de proteína e decréscimo na excreção de nitrogênio (N) via ureia (Hornick et al., 1998), o que fica evidente no grupo PLCO no Período 1, momento este ao qual os animais passaram pela primeira prova de laço comprido. Segundo os mesmos autores, pode ocorrer o aumento da retenção de $\mathrm{N}$ como resultado da melhora na digestibilidade e aumento da eficiência de sua metabolizabilidade.

Tabela 1. Níveis séricos de ureia, creatinina e proteína total (PtnT) dos grupos, sem atividade (SEMA), prova de laço comprido (PLCO) e treino (TREI).

\begin{tabular}{lcccc}
\hline & & Grupos & \\
\hline Período 1 & SEMA & PLCO & TREI & EPM \\
\hline Ureia (mg/dL) & $29,29^{\mathrm{aA}}$ & $13,57^{\mathrm{c}}$ & $21,43^{\mathrm{b}}$ & 1,62 \\
PtnT (g/dL) & $7,18^{\mathrm{B}}$ & $7,01^{\mathrm{C}}$ & $7,07^{\mathrm{B}}$ & 0,17 \\
Creatinina (mg/dL) & $2,44^{\mathrm{aA}}$ & $2,54^{\mathrm{aA}}$ & $2,13^{\mathrm{b}}$ & 0,12 \\
\hline Período 2 & & & 1,64 \\
\hline Ureia (mg/dL) & $14,83^{\mathrm{B}}$ & 14,12 & 0,00 & 0,17 \\
PtnT (g/dL) & $7,58^{\mathrm{B}}$ & $7,55^{\mathrm{B}}$ & $7,13^{\mathrm{B}}$ & 0,12 \\
Creatinina (mg/dL) & $1,72^{\mathrm{B}}$ & $1,70^{\mathrm{B}}$ & 1,73 & \\
\hline Período 3 & & & 16,85 & 1,62 \\
\hline Ureia (mg/dL) & $15,00^{\mathrm{B}}$ & 16,86 & $8,31^{\mathrm{A}}$ & 0,17 \\
PtnT (g/dL) & $8,26^{\mathrm{A}}$ & $8,04^{\mathrm{A}}$ & 1,86 & 0,12 \\
Creatinina (mg/dL) & $1,91^{\mathrm{B}}$ & $1,94^{\mathrm{B}}$ & & \\
\hline
\end{tabular}

Letras maiuscúlas diferentes nas colunas e letras minuscúlas diferentes nas linhas diferem estatisticamente $(\mathrm{P}<0,05)$ entre si pelo teste de Scott Knott. EPM= Erro padrão da média.

Período 1, análise realizada em maio de 2016 (após a primeira prova de laço comprido); Período 2, análise realizada em agosto de 2016 (não houve competição); e Período 3, análise realizada em dezembro de 2016 (após a segunda prova de laço comprido).

Ainda, a concentração de nitrogênio uréico no sangue está relacionada com o catabolismo/anabolismo de aminoácidos, o qual também é influenciado pela restrição alimentar e ingestão de proteína da dieta, respectivamente (Ellenberger et al., 1989). Provavelmente a redução nas concentrações de ureia nos animais do grupo PLCO e TREI, comparativamente ao grupo SEMA, pode estar associado ao tempo que o animal ficava em jejum durante a competição e o treino, respectivamente, no entanto, isso só ocorreu no Período 1. Uma provável hipótese para o ocorrido pode ter sido a adaptação dos animais as atividades físicas aos quais eram submetidos, a partir das segundas avaliações.

Segundo Kaneko et al. (1997) os valores de ureia no plasma bovino estão entre 17 e $45 \mathrm{mg} / \mathrm{dL}$ aproximadamente. Os animais do grupo PLCO apresentaram valores abaixo em ambos os períodos, enquanto que o grupo SEMA com valores considerados adequados apenas no Período 1, diferindo estatisticamente do Período 2 e 3. Apesar da redução dos níveis de ureia ao longo do projeto nos animais do grupo SEMA, tal fato não pode ser associado ao ganho ou crescimento compensatório, já que os mesmos foram mantidos sobre as mesmas condições de manejo e pastejo ao longo de todo o projeto.

A creatinina é um composto nitrogenado produzido a partir da fosfocreatina muscular. A quantidade de creatinina formada por dia depende da quantidade de creatina no organismo, que por sua vez depende da massa muscular. Assim, de acordo com os dados desse trabalho, a atividade física e a restrição alimentar sofrida pelos bovinos jovens do grupo PLCO não foram suficientes para redução da massa muscular no Período 1, baseando-se nas concentrações de creatinina, já que os animais do grupo SEMA apresentaram a mesma concentração, não diferindo nos demais. De acordo com Keogh et al. (2015), animais submetidos a restrição alimentar é observado catabolismo proteico, e consequente, com a redução da massa corporal há aumento na concentração plasmática de creatinina.

Não foi observada diferença significativa do eritrograma dos animais submetidos à prova de laço comprido, independente da prova ou do momento de coleta do sangue (antes ou após o término da prova no mesmo dia) (Tabela 2). No entanto, cabe frisar que os valores para hematócrito e hemoglobina são semelhantes aos valores médios 
de 37,50 e $11,91 \%$, respectivamente, dos animais criados em sistema extensivo, encontrados por Silva et al. (1992).

Segundo Ferreira (2010), o aumento da concentração de hematócrito após a corrida pode estar relacionado com o exercício físico juntamente com a falta da ingestão de água, somado a isso os animais ao fim da prova podem apresentar-se fatigados e cansados, entretanto, não houve aumento na concentração de hematócrito neste estudo. A diferença numérica ocorrida neste estudo entre os indivíduos ativos na situação de repouso em comparação ao final do exercício está provavelmente relacionado apenas ao aumento da viscosidade sanguínea, ou seja, desidratação, não sendo uma variação suficiente para um efeito estatístico.

Em cavalos submetidos ao teste de simulação da prova de Team Penning o atributo mais importante na análise de sangue é a quantidade de glóbulos vermelhos conhecidos como hemácias (Miranda et al., 2011). De acordo com estes autores houve aumento na concentração das hemácias e das concentrações de hemoglobina imediatamente após o exercício físico em cavalos, resultados diferentes dos encontrados nesse estudo, entretanto com bovinos. Tal resultado pode estar associado à maior adaptação dos equinos a atividade muscular intensa, desenvolvendo mecanismos que mantenha o alto desempenho durante um período maior, já que a hemoglobina (componente das hemácias) está associada ao transporte de oxigênio. De maneira geral, os valores da concentração da hemoglobina (11,65 e $12,58 \mathrm{mg} / \mathrm{dL}$ ) estão dentro da média descritas por Fagliari et al. (1998) em animais adultos da raça Nelore criados em sistemas extensivos $(9,93 \mathrm{e}$ $15,29 \mathrm{mg} / \mathrm{dL}$ ).

Tabela 2. Análises hematológicas em bovinos antes e após à atividade em provas de laço comprido.

\begin{tabular}{|c|c|c|}
\hline \multirow[b]{2}{*}{ Características } & \multicolumn{2}{|c|}{ Primeira Prova } \\
\hline & Antes & Após \\
\hline Hematócrito $(\%)$ & 37,94 & 39,77 \\
\hline Hemácias (milhões $/ \mathrm{mm}^{3}$ ) & 8,56 & 8,57 \\
\hline Hemoglobina (g/dL) & 11,91 & 12,64 \\
\hline $\operatorname{VCM}(\%)$ & 44,28 & 46,51 \\
\hline $\operatorname{HCM}(\%)$ & 13,91 & 14,75 \\
\hline \multirow[t]{2}{*}{$\mathrm{CHCM}(\%)$} & 33,44 & 31,83 \\
\hline & \multicolumn{2}{|c|}{ Segunda Prova } \\
\hline Características & Antes & Após \\
\hline Hematócrito $(\%)$ & 37,27 & 40,3 \\
\hline Hemácias (milhões/mm³) & 8,39 & 8,56 \\
\hline Hemoglobina $(\mathrm{g} / \mathrm{dL})$ & 11,65 & 12,58 \\
\hline $\operatorname{VCM}(\%)$ & 44,37 & 47,03 \\
\hline $\operatorname{HCM}(\%)$ & 13,8 & 14,71 \\
\hline $\mathrm{CHCM}(\%)$ & 31,34 & 31,31 \\
\hline
\end{tabular}

A restrição alimentar e a exigência física dos animais reduziram o ganho médio diário $\mathrm{e}$ consequentemente a média final de peso vivo dos animais do grupo TREI (Tabela 3), tal fato pode estar associado ao tempo desprendido pelos animais semanalmente para treinamento dos peões. A perda de peso resultou na mobilização de um compartimento protético muito lábil, o qual corresponde à proteína recentemente depositada. A quantia de proteína lábil depositada no corpo do animal não excede $5 \%$ do total de proteína corporal e desaparece dentro de alguns dias quando o animal está em restrição alimentar ou alimentado com uma dieta com déficit de proteína (Paquay et al., 1972).

De acordo com Mitra et al. (1972) e Encarnação (1986), um organismo em situação de estresse, a hipófise secreta menos hormônio somatotrófico ou de crescimento, além de menos hormônios tireotróficos e gonadotróficos, como hormônio folículo estimulante e hormônio luteinizante, conduzindo, os dois últimos, a uma reduzida atividade da tireóide e das gônadas. Assim, dado o efeito catabólico estimulado pelos glicocorticosteróides, pode ocorrer constante degradação de tecidos musculares e adiposos, 
assim como a inibição da síntese desses tecidos, provocando a perda de peso e crescimento reduzido, que se observou nos animais do grupo TREI, que apresentaram o menor desenvolvimento corporal, comparativamente aos demais grupos avaliados, provavelmente devido ao maior estresse acometido ao grupo.

Tabela 3. Médias das análises biométricas e peso vivo final (PVF) dos grupos, sem atividade (SEMA), prova do laço comprido (PLCO) e treino (TREI).

\begin{tabular}{|c|c|c|c|c|c|}
\hline \multicolumn{6}{|c|}{ Grupos } \\
\hline Características & SEMA & PLCO & TREI & Valor de $P$ & EPM \\
\hline $\mathbf{A A}$ & $132,50^{\mathrm{a}}$ & $135,30^{\mathrm{a}}$ & $122,50^{\mathrm{b}}$ & $<0,01$ & 0,61 \\
\hline $\mathbf{A P}$ & $140,17^{\mathrm{b}}$ & $142,26^{\mathrm{a}}$ & $131,14^{\mathrm{c}}$ & $<0,01$ & 0,66 \\
\hline PTO & $63,28^{\mathrm{a}}$ & $64,29^{\mathrm{a}}$ & $57,42^{\mathrm{b}}$ & $<0,01$ & 0,50 \\
\hline PT & $168,34^{\mathrm{b}}$ & $171,95^{\mathrm{a}}$ & $156,15^{\mathrm{c}}$ & $<0,01$ & 1,14 \\
\hline $\mathrm{CC}$ & $141,03^{\mathrm{a}}$ & $143,78^{\mathrm{a}}$ & $127,01^{b}$ & $<0,01$ & 1,14 \\
\hline CG & $41,18^{\mathrm{a}}$ & $42,16^{\mathrm{a}}$ & $37,21^{\mathrm{b}}$ & $<0,01$ & 0,35 \\
\hline LGI & $39,12^{\mathrm{b}}$ & $40,22^{\mathrm{a}}$ & $35,15^{\mathrm{c}}$ & $<0,01$ & 0,33 \\
\hline LGIS & $12,98^{\mathrm{b}}$ & $13,41^{\mathrm{a}}$ & $12,06^{\mathrm{c}}$ & $<0,01$ & 0,12 \\
\hline ASE & $68,91^{\mathrm{a}}$ & $68,36^{\mathrm{a}}$ & $64,95^{\mathrm{b}}$ & $<0,01$ & 0,37 \\
\hline PVF & $329,93^{\mathrm{a}}$ & $348,15^{\mathrm{a}}$ & $306,10^{\mathrm{b}}$ & $<0,01$ & 6,61 \\
\hline
\end{tabular}

Letras minuscúlas diferentes nas linhas diferem estatisticamente $(\mathrm{P}<0,05)$ entre si pelo teste de Scott Knott.

AA: Altura do Anterior; AP: Altura do Posterior, PTO: Profundidade Torácica; PT: Perímetro Torácico; CC: Comprimento Corporal; CG: Comprimento de Garupa; LGI: Largura da garupa entre os Ílios; LGIS: Largura da garupa entre os Ísquios; ASE: Altura Sub Esternal (ASE); EPM: Erro padrão da média.

Ademais, o aumento da exigência energética de animais submetidos ao exercício físico está associado, também, à redução no peso corporal e ganho médio diário (Sejian et al., 2012). Ryan (1990) ainda afirma que há uma variabilidade das respostas do crescimento compensatório, tendo em vista os diversos fatores que podem estar envolvidos na manifestação do fenômeno.

De acordo com Restle et al. (2006) animais compridos e profundos (profundidade torácica) são desejados em sistemas de terminação e que, consequentemente, serão mais pesados e depositarão maior quantidade de gordura de cobertura, entretanto, tal avaliação não foi observada nos animais do grupo TREI. De maneira geral, as medidas variam em função do crescimento esquelético, atingindo um limiar à maturidade, enquanto que o peso e a circunferência torácica variam em função do crescimento muscular (Prajapati et al., 1991), que também foram menores nos animais do grupo TREI.

\section{Conclusão}

A creatinina e a ureia não permitiram analisar alterações fisiológicas em bovinos submetidos a prova de laço comprido.

O desenvolvimento corporal foi maior nos animais que sofreram o estímulo muscular durante a prova de laço comprido, porém, a elevada exigência física dos animais do grupo treino, provocaram retardamento no crescimento.

\section{Conflito de Interesse}

Os autores declaram não existir conflito de interesse.

\section{Comitê de Ética}

A Comissão de Ética no Uso de Animais (CEUA)- Universidade Estadual de Mato Grosso do Sul (UEMS) certificou a utilização dos animais de acordo com o protocolo $\mathrm{n}^{\circ}$ 020/2016.

\section{Referências}

Bullock, K.D.; Bertrand, J.K.; Benyshek, L.L. Genetic and environmental parameters for mature weight and other growth measures in Polled Hereford cattle. Journal of Animal Science, 71(7): 1737-1741, 1993.

Camargo, F.G.; Teixeira Neto, A.R.; Freitas D.F.H.; Lacerda Neto, J.C.; Queiroz-Neto, A. Alterações hematológicas e cardíacas em cavalos Árabes submetidos ao teste de esforço crescente em esteira rolante. Brazilian Journal of Veterinary Research and Animal Science, 46(6): 431-437, 2009.

D’Oliveira, M.C.; Souza, M.I.L.; Filho Corrêa, R.A.; Morais, M.G.; Ítavo, C.C.B.F.; Franco, G.L. Effects of road transportation or droving 
on the weight and metabolism of young bulls. Tropical Animal Health and Production, 46(8): 1447-1453, 2014.

Ellenberger, M.A.; Johnson, D.E.; Carstens, G.E.; Hossner, K.L.; Holland, D.; Nett, T.M.; Nockels, C.F. Endocrine and Metabolic Changes during Altered Growth Rates in Beef Cattle. Journal of Animal Science, 67(6): 1446-1454, 1989.

Encarnação, R.O. Estresse e produção Animal. In: EMBRAPA-CNPGC. Documentos,

Campo Grande, 1986. p.32. 1986.

Fagliari, J.J.; Santana, A.E.; Lucas, F.A.; Campos Filho, E.; Curi, P.R. Constituintes sanguíneos de bovinos lactantes, desmamados e adultos das raças Nelore (Bos indicus) e Holandesa (Bos taurus) e de bubalinos (Bubalus bubalis) da raça Murrah. Arquivo Brasileiro de Medicina Veterinária e Zootecnia, 50(3): 263-271, 1998.

Ferreira, F.G.; Alves, K.; Costa, N.M.B.; Santana, A.M.C.; Marins, J.C.B. Efeito do nível de condicionamento físico e da hidratação oral sobre a homeostase hídrica em exercício aeróbico. Revista Brasileira de Medicina do Esporte, 16(3): 166-70, 2010.

González, F.H.D.; Conceição, T.R.; Siqueira, A.J.S.; La Rosa, V.L. Variações sanguíneas de uréia, creatinina, albumina e fósforo em bovinos de corte no Rio Grande do Sul. A Hora Veterinária, 20(1): 59-62, 2000.

Hornick, J.L.; Van Eenaeme, C.; Clinquart, A.; Diez, M.; Istasse, L. Different periods of feed restriction before compensatory growth in Belgian Blue bulls: I. animal performance, nitrogen balance, meat characteristics, and fat composition. Journal of Animal Science, 76(1): 249-259, 1998.

Kaneko, J.J.; Harvey, J.W.; Bruss, M.L. Clinical biochemistry of domestic animals. $5^{\text {th }} \mathrm{ed}$. New York: Academic Press, 1997. 932p.

Keogh, K.; Waters, S.M.; Kelly, A.K.; Kenny, D.A. Feed restriction and subsequente realimentation in Holstein Friensian bulls: I. Effecton animal performance; muscle, fat, and linear body measurements; and slaughter characteristics. Journal of Animal Science, 93(7): 3578-3589, 2015.

Lachica, M.; Prieto, C.; Aguilera, J.F. The energy costs of walking on the level and on negative and positive slopes in the Granadina goat (Capra hircus). The British journal of nutrition, 77(1): 73-81, 1997.
Miranda, R.L.; Mundim, A.V.; Saquy, A.C.S.; Costa, Á.S.; Guimarães, E.C.; Gonçalves, F.C.; Carneiro, F.O. Perfil hematológico de equinos submetidos à prova de Team Penning. Pesquisa Veterinária Brasileira, 31(1): 81-86, 2011.

Mitra, R.; Christison, G.I; Johnson, H.D. Effects of prolonged thermal exposure on growth hormone $(\mathrm{GH})$ secretion in cattle. Journal of Animal Science, 34(5): 776-779, 1972.

Pacheco, A.; Quirino, C.R.; Pinheiro, O.L.V.M.; Almeida, J.V.D.C. Medidas morfométricas de touros jovens e adultos da raça Guzerá. Revista Brasileira de Saúde e Produção Animal, 9(3): 426-435, 2008.

Paquay, R.; De Baere, R.; Lousse, A. The capacity of the mature cow to lose and recover nitrogen and the significance of protein reserves. British Journal of Nutrition, 27(1): 27-37, 1972.

Prajapati, K.B.; Radadia, N.S.; Tajane, K.R. Relationship between different measures of body size in Mehsana buffaloes. Indian Journal of Animal Science, 61(1): 88-90, 1991.

R Development Core Team. R: A language and environment for statistical computing. Vienna: R Foundation for Statistical Computing, 2008.

Restle, J.; Pacheco, P.S.; Alves Filho, D.C.; Freitas, A.K.; Neumann, M.; Brondani, I.L.; Pádua, J.T.; Arboitte, M.Z. Silagem de diferentes híbridos de milho para produção de novilhos superjovens. Revista Brasileira de Zootecnia, 35(5): 2066-2076, 2006.

Ryan, W.J. Compensatory growth in cat the and sheep. Nutrition Abstracts and Reviews, 50(9): 653-664, 1990.

Sejian, V.; Maurya, V.P.; Kumar, K.; Naqvi, S.M.K. Effect of multiple stresses on growth and adaptive capability of Malpura ewes under semi-arid tropical environment. Tropical Animal Health and Production, 45(1): 107116, 2013.

Sejian, V.; Maurya, V.P.; Naqvi, S.M.K. Effect of walking stress on growth, physiological adaptability and endocrine responses in Malpura ewes in a semi-arid tropical environment. International Journal of Biometeorology. 56(2): 243-252, 2012.

Silva, M.B.; D'Angelino, J.L.; Araujo, W.P.; Galhardo, M.; Garcia, M.; Birgel, E.H. Avaliação do eritrograma de búfalos (Bubalus bubalis) criados na região do Vale do Ribeira em São Paulo. Brazilian Journal of 
Veterinary Research and Animal Science, 29(1): 113-119, 1992.

Van Eenaeme, C.; Evrard, M.; Hornick, J.L.; Baldwin, P.; Diez, M.; Istasse, L. Nitrogen balance and myofibrillar protein turnover in double muscled Belgian Blue bulls in relation to compensatory growth after different periods of restricted feeding. Canadian Journal of Animal Science, 78(4): 549-559, 1998. 